\title{
Fine-Needle Aspiration Cytology Is an Alternative Source of High Quality Archival Samples in Biobanking
}

\author{
Sani Abubakar Malami ${ }^{1,2}$ \\ ${ }^{1}$ Faculty of Medicine, Bayero University, P.M.B. 3011, Kano 700001, Nigeria \\ ${ }^{2}$ Department of Histopathology, Aminu Kano Teaching Hospital, P.M.B. 3452, Kano 700001, Nigeria
}

Correspondence should be addressed to Sani Abubakar Malami, malamisa@yahoo.co.uk

Received 5 May 2011; Accepted 3 June 2011

Academic Editors: J. A. Jimenez-Heffernan and K. K. Khurana

Copyright ( $) 2011$ Sani Abubakar Malami. This is an open access article distributed under the Creative Commons Attribution License, which permits unrestricted use, distribution, and reproduction in any medium, provided the original work is properly cited.

\begin{abstract}
Advances in fine-needle aspiration cytology (FNAC) techniques allows for a whole range of procedures on these limited sources of tissue. Where fresh aspirates are not readily available, archival FNAC samples had also been used for clinical diagnoses, molecular profiling, and disease monitoring with impressive results. Nonetheless, legitimate concerns exist regarding the reliability of these banked specimens. Increased demands on human tissues in response to the rapid acceleration on emerging scientific platforms will probably make tissue bank creation derived from archival FNAC a priority. To highlight its future prospects as a resource for tissue banking, this paper provides an overview of the use of archival FNAC in clinical situations and ancillary studies. It is concluded that tissue banks of archival FNAC specimens might have great promise in optimizing patient care and translational research.
\end{abstract}

\section{Introduction}

Tissue banking refers to a chain of activities directed at the collection, storage, and distribution of residual specimens of abnormal and normal human tissues [1]. Accordingly, it encompasses a wide range of specimen types and sample collection designs, ranging from population-based banking of normal tissues to specific biobanking of diseased specimens obtained in the course of clinical interventions and autopsies [1-3].

Tissue banking is a central activity in virtually all aspects of biomedical research and progress in the development of personalized medicine $[2,4]$. In a recent article, $\mathrm{Yu}$ and Zhu stated that tissue bank creation has emerged as a global priority in view of the rapid acceleration on new technological platforms which had increased the demands on tissues and peripheral blood for research at the DNA, mRNA, and protein levels [5].

Considerable advances in biomedical research had been achieved on high-quality samples derived from large series of cases from well-organized archival FFPE tissue banks.
Also cryopreserved samples and tumour tissue imprint form a significant proportion of biobanked tissues. Among cytological preparations, the potential value of archival cervical smears, especially, had been highlighted [6].

Clearly, the search is on for an inexhaustible source of high-quality pathological samples with matching clinical data for cutting-edge research. To explore a possible role for archival FNAC-based tissue banks in the emerging scenario, this paper examines the evidence base in major bibliographic databases. A brief systematic literature review of international peer-reviewed literature was undertaken using Medline. Grey literature was identified through generic search engines. The search period was from 1 January 1996 to 30 November 2010. The search criteria were English language manuscripts and search terms "fine-needle aspiration cytology," "tissue banks," "cytological diagnosis," and "archival" as a major heading. Boolean operators were used to combine the search terms. Identified abstracts were independently reviewed, and the findings are presented as a narrative summary. 


\section{Limitations of Archival Paraffin-Embedded Tissues}

Routinely stored archival, formalin-fixed, and paraffinembedded (FFPE) tissues had been the mainstay of retrospective studies for diagnostic and prognostic purposes. In molecular studies, stored paraffin blocks allow for obtaining RNA, though with features of considerable degradation [7]. In previous studies of banked human tissues, it had been shown that the reliability of results obtained from tissue analysis is a function of specimen quality, which may be affected by conditions that may contribute to the degradation of the specimen due to its prior storage [7]. These specimens also have been noted to have serious limitations in terms of technical difficulties related to microdissection and poor DNA quality [8]. Among cytological specimens, PCR-based DNA testing of archival cervical smears has also been popularized, but the original fixation method applied on the sample might play a significant role in the eventual success or other wise of recovery of DNA material [9].

There is thus an unmet clinical need for economic, relatively noninvasive procedures that use a limited number of cells for medical research. The growing need for fast, highly sensitive and quantitative technologies for molecular characterization of scant cells in biological samples derived from easily accessible bodily sources including fine-needle aspirates will have significant impact in life sciences and clinical practice [10].

\section{Potential of Archival FNAC in Tissue Analysis}

Fine-needle aspiration cytology (FNAC) is a minimally invasive, cost-effective, and highly sensitive tool for presurgical differential diagnosis. FNAC has the distinct advantage that samples can be obtained on multiple occasions conveniently. It goes without saying that the process of acquiring samples are, in any event, a lot more pleasant to the patient than, say, excision biopsy. A further advantage is that the reliability of fresh FNAC samples for rendering a specific diagnosis can be improved by applying several ancillary techniques [11]. However, sometimes critical decisions on patient care may need to be made when fresh tissue is not available or when the cytologic diagnosis needs further clarification. In these situations, slide scrape lysates (SSL) of archival cytologic material could be utilized [12]. In contrast to fresh samples, archival FNAC specimens, offer the chance for a careful morphologic review and interpretation prior to molecular analysis. This allows the trained cytologist to preselect cases and slide preparations for subsequent molecular analysis, resulting in optimal utilization and cost control in the molecular laboratory [12]. Despite this huge potential of archival FNAC samples, only rarely are they utilized for molecular tests in routine practice. Additionally, until recently, FNAC samples were rarely archived, and it took many months to accumulate enough specimens for a study [13].

\section{Tissue Acquisition by the Slide Scrape Extraction Procedure}

Archival FNAC samples can be processed by the slide scrape lysates procedure (SSL) when the fresh sample is insufficient for patient monitoring or too difficult to interpret. A number of workers have described the preparation of the slide scrape lysates from archival FNAC $[8,12,14,15]$. Briefly, coverslips are detached from the slides via immersion in xylene, and the slides are hydrated in a graded series of alcohol, followed by soaking in distilled water for 2 minutes. Following this, the slides are hydrated in a graded series of ethanol, followed by immersion in distilled water for 1 minute. The slides are subsequently allowed to air-dry completely. Then using a diamond-tipped pencil or pen, the area of interest (area of atypical cells) on the archival FNA slide is marked. The diamond-tipped pencil is used to mark the underside of the slide. Using the area of interest indicated by the marking on the FNA slide as a guide, slide scrape lysates (SLL) are prepared by using a single-edged razor blade to scrape the areas of the slide containing the atypical cells of interest. A volume of $20 \mathrm{~mL}$ of digestion buffer solution $(50 \mathrm{mM}$ Tris, $1 \mathrm{mM}$ EDTA, $0.5 \%$ Tween $\mathrm{pH} 8.0$ ) is pipetted onto the areas of the slide previously marked with diamond pencil. These areas are then carefully scraped with a sterile single edge razor blade (or scalpel) to dislodge cells from the slide surface. At this point the mixture of scraped cells and buffer can be pipetted into a microcentrifuge tube, ready for use.

\section{Role of Archival FNAC in Oncology}

Molecular diagnostic cytopathology is in itself a comparatively new field. A small series have given detailed descriptions of the utilization of SSLs of cytologic samples [16-18]. A review of the literature showed that archival cytologic samples and FNAC, in particular, may be suitable for manipulation in molecular tests $[6,12,16,19-21]$. Among other things, the ability to use archival cytology slides greatly increases the potential material available for molecular analysis in the selection of patients for targeted therapeutic agents [22], and this principle may be extended to tests on remotely obtained samples. Nonetheless, most of the molecular tests using FNAB specimens are currently investigational [11].

The polymerase chain reaction (PCR) is a molecular test that has an established role in the diagnosis of cancer, infectious diseases, and genetic disorders. It enables the amplification of specific sequences of nucleic acids from an extremely small amount of genetic material. Ordinarily, the PCR test is performed on fresh specimens, but it had been shown that archival cytologic samples may also be suitable for it.

In one of such previous works, SSL of 13 existing FNAs were among 112 samples that were evaluated for amplification status compared to a randomly chosen control group of equivalent cytologic samples submitted fresh. 85\% had amplifiable DNA, as assessed by a positive reaction for glyceraldehyde phosphate dehydrogenase (GAPDH), and 
there was no statistical difference in the amplification yield between both the test and control groups $(P=0.2177)$. This goes to show that even if fresh tissue is not available, critical decisions on patient care can still be made by molecular diagnostic tests carried out on archival FNA materials [12].

Prior to the work of Liu et al., archived FNAC cytologic materials had been rarely investigated for their suitability for gene expression analysis. The authors addressed this issue by reverse transcription and polymerase chain reaction (RTPCR) of the glucose-6-phosphate dehydrogenase (G6PD) transcript in a series of archival histologic and cytologic specimens [23]. They found that neither staining nor prolonged storage up to 15 years had major negative effects on RT-PCR and concluded that these samples are valuable resources for RT-PCR-based molecular investigations.

Another work published recently focused on the use of the PCR for the detection of B- and T-cell clonality on archival FNA samples. The aim of that study, by Štoos-Veić et al. [24], was to determine the frequency of PCR detection of IgH/BCL2 in DNA samples isolated from archival cytological slides of lymph node aspirates, bone marrow, and/or peripheral blood $(\mathrm{PB})$ obtained from patients with histologically confirmed follicular lymphoma. They successfully isolated DNA from all archival cytological slides obtained by fine-needle aspiration of lymph nodes. Fusion oncogene was detected in 10 of 19 patients (52\%). Among patients with PCR IgH/BCL2 positive lymph nodes, molecular test found $\mathrm{BM}$ infiltration in 5 cases. The implication is that the rate of detection of IgH/BCL2 rearrangement in FNA specimens is similar to results reported for paraffin-embedded tissues [24].

To optimise molecular analysis of archival tissues, it is essential that fixation preserves morphology along with protein epitopes and DNA/RNA integrity [11]. Kube and others [25] conducted a study that involved thirty patients who had FNA of lymphoid tissue collected both retrospectively and prospectively. The DNA was amplified with primers specific for the major break point region of the $t(14 ; 18)$ translocation, and the presence of the bcl-2 oncogene was correlated with clinical, cytomorphologic, histologic, and immunologic findings. The cell suspensions provided sufficient DNA for PCR amplification, and thus the method detected the bcl-2 oncogene in various cytologic lymphoid smears, both archival and fresh [25].

Until quite recently, it had been unclear whether biomarkers such as c-myc amplification could be analyzed on genetic materials collected from archival fine-needle aspiration (FNA) smears. A novel comparative PCR analysis was used to analyze c-myc amplification semiquantitatively, and this suggested that c-myc amplification may be a biomarker of breast cancer risk [26].

Some of the more recent works on archived and stained fine-needle aspirate smears have sought to highlight the fact that these materials can be used for clinical molecular diagnosis and profiling. Moreover, that cytopathology specimens have been found to be an excellent potential source of patient materials and FNAC samples up to 16 years old yielded copious quantities of genomic DNA suitable for high-resolution genomic and epigenomic profiling. This was the conclusion of a study performed at the National Cancer Institute, (Bethesda, Md, USA), in which 57 fine-needle aspiration cytopathology (FNAC) samples were examined for aberration detection on high-resolution comparative genomic hybridization array, DNA methylation, and single nucleotide polymorphism genotyping platforms [27].

Mutational analysis and/or gene copy number is being increasingly used in choosing among a wide range of targeted therapies for nonsmall cell lung cancer (NSCLC) due to the discovery that somatic mutations in the epidermal growth factor receptor (EGFR) gene are associated with sensitivity to the EGFR tyrosine kinase inhibitors (TKIs) in lung adenocarcinomas, whereas KRAS mutations are associated with resistance [28]. In this regard, Smith et al. [15] used eleven archival fine-needle aspirate (FNA) specimens from advanced NSCLC patients in a study of EGFR mutation in which all DNA samples were successfully amplified by PCR. EGFR mutation status was determined successfully using a slide-scrape DNA extraction protocol of selected tumour cell regions on the smear slides, followed by realtime PCR and high-resolution melt analysis (HRMAA) of EGFR exons 18, 19, 20, and 21, followed by sequence analysis. Furthermore, the clinical utilization of fresh FNA cytology specimens as a reliable source for molecular testing to detect KRAS mutations in patients with NSCLC has been validated recently. Among 99 patients with lung cancer who had FNA, DNA sequencing for EGFR exons 18-21 and KRAS codons 12,13 , and 61 was performed [29]. KRAS mutations were detected in $23.6 \%$ (41 of 174) of NSCLCs with no statistical differences between adenocarcinoma (26\%, 26 of 102) and nonadenocarcinoma $(21 \%, 17$ of $72, P=0.86)$.

In recognition of the fact that follicular variant of papillary thyroid carcinoma is a lesion that frequently causes difficulties from a diagnostic perspective in the laboratory, Denning et al. applied a panel of markers using real-time PCR on archival thyroid FNA to determine their utility or otherwise as adjunctive markers in the diagnosis of this variant of thyroid carcinoma. The panel comprised HLADMA, HLA-DBQ1, CD74, CSNK1G2, IRF3, KRAS2, LYN, MT1K, MT1X, RAB23, TGFB1, and TOP2A, with CDKN1B as an endogenous control. They found that this panel of molecular targets discriminates between follicular adenoma, papillary thyroid carcinoma, follicular variant of papillary thyroid carcinoma, and follicular thyroid carcinoma by their expression repertoires [30].

Evaluation of HER-2 status is routinely done on FFPE tissues, but few studies have analyzed this marker on stored FNAC specimens. In one of such studies, a new method was used with an automated detection system to demonstrate that FISH is feasible on archival MGG-stained slides and is also a reliable option for assessing HER-2 status [31].

There are still some ongoing studies that could open a new vista in diagnostic molecular cytopathology as far as archival FNA is concerned. Worth mentioning is a recent work that employed mutational analyses by polymerase chain reaction (PCR), single-strand conformational polymorphism analysis (SSCP), and sequencing for p53 exons 58 in breast lesions with indeterminate or suspicious cytology as a diagnostic criterion on archival FNA specimens. This 
study produced rather inconclusive results and awaits the conduct of validation studies [32].

Whereas technologies in current clinical use are often time consuming, expensive, or require considerable sample sizes, analysis of materials derived from archival FNAC tissue banks is a most sensitive technique to detect and profile unaltered cells and promises to play a critical role in the future in the discovery of molecular patterns of cancers and for the implementation of novel biomarkers into clinical trials.

\section{Archival FNA in Infectious Disease}

A pioneering work on the efficacy of archival FNA in molecular diagnostic cytopathology of infections was published in 1999. It describes a study that sought to demonstrate that the accuracy of Kaposi's Sarcoma diagnosis can be improved by FNA, in which 13 spindle-cell lesions obtained by FNA were analyzed for the presence of HHV-8 DNA [33]. The researchers used DNA isolated from archival WrightGiemsa-stained glass slides for PCR amplification of the HHV-8 DNA sequences. All of the cases diagnosed as KS were PCR-positive for HHV-8 DNA.

Polymerase chain reaction (PCR) for Mycobacterium tuberculosis complex is useful on routinely stained cytologic samples from patients with extrapulmonary tuberculosis. It can be reliably performed on archival cytological slides from extrapulmonary samples and is highly sensitive, though it may lead to positive results in immunocompromised patients without any sign of active tubercular disease [34]. However, detection of $M$. tuberculosis (MTB) in thyroid lesions may be notoriously difficult with routine cytological and acid-fast bacilli (AFB) staining. In a recent article, Gupta et al. described a technique for extraction of DNA from limited archival FNAC material available on May-GrünwaldGiemsa-stained smears by PCR performed for the insertion sequence IS6110 for MTB [35].

Reverse transcription-polymerase chain reaction (RTPCR) is a foremost tool for measuring gene expression profiles in biological samples. Notably, studies have demonstrated the PCR-based DNA testing of archival cervical smears. This can be used retrospectively to establish the presence of human papilloma virus [9]. It remains to be seen whether this technique would be equally applicable and useful in translational research on archival FNA samples derived from other HPV-related cancers particularly those of the head and neck region.

\section{Miscellaneous Applications}

7.1. Quantitative Techniques and Archival Fine-Needle Aspiration Specimens. DNA methylation is a common event in many cancers, and it seems certain that the changes in the methylation patterns can play an important role in tumorigenesis. This underscores the reason why the evaluation of the methylation status of several genes in various tissue types is important. The successful elucidation of methods by which nucleic acids derived from archived FNAC samples could be used in various gene expressions, gene amplification studies, and for the validation of biomarkers had been quite a challenging task. Recently, Sahoo and others developed a method which improved substantially, the previously published protocol in terms of yield, for a more sensitive and specific method for determination of methylation status of multiple genes of chromosomal DNA and the promoter region in archived specimens FFPE and fine-needle aspirates [36]. Thus, the group was able to demonstrate that the limited amount of cells available from the FNAC smears could be used for the evaluation of the methylation status of various genes postmodification.

In a work published by Billah et al. [29], the novel reverse-phase protein microarray (RPPM) technology was applied successfully for the quantitative analysis of breast FNAC specimens. It was demonstrated from this study that low-abundance analytes and phosphorylated and nonphosphorylated proteins in specimens that consist of a few thousand cells obtained through FNAC can be quantified with RPPM technology. The authors recommend this technique for its ability to monitor the in vivo state of cell-signaling proteins before and after treatment saying that it would potentially augment further the development of rational drug targets.

There are also many potential diagnostic applications of proteomics to archival FNAC specimens which would allow for rapid, reproducible protein profiling directly from crude samples. For instance, Fetsch et al. [37] had used SELDI analysis (surface-enhanced laser desorption/ionization spectrometry) to highlight the differences in protein expression profiles in a series of cases directly from FNA cytologic samples. This would be potentially useful in the early diagnosis and treatment of cancers, subject to the result of larger scale studies and further characterization of these proteins. In the opinion of the authors, the use of archival cytologic specimens for these purposes will be quite appealing for various reasons. The most important reason being its potential to enable unique protein spectra to be linked to specific prognostic or outcome data, including rapidity of disease course and specific therapy susceptibility which would be particularly appealing when only limited numbers of cells are available, such as in fine-needle aspirates.

\section{Conclusion}

There is a rising demand for human samples needed to further advance our knowledge of disease development, thus leading to better prevention and treatment strategies for cancers, infections, and other disorders. Since an easier access to these samples for the scientific community is considered as the main bottleneck for research for health, this dictates that specimens must be sourced from distinct forms of biobanking [38]. FNAC is a technique that is well accepted by the patient and clinicians alike and archival specimens derived therefrom could be a principal resource for medical research. Stored FNAC samples are gaining increasing importance as rapid and simple method for obtaining DNA samples and could represent a vast untapped resource for clinical diagnoses and molecular investigations. 
In selected cases, the application of molecular markers to archival FNAC will help to prevent unnecessary surgeries and guide targeted therapies.

FNAC has not adequately contributed to the current efforts to establish large and well-organized biobanks. To accomplish the current requirements of the scientific community, archival FNAC tissue banks, like all other biobanks, need to address some essential challenges that include management strategies, consensus on standard operating procedures, appropriate design, harmonized and unique policies of utilization, and sustainability, all of them within the framework of the appropriate ethical, legal, and social considerations [2].

More research would be needed in the future to determine the impact of FNAC tissue banking on the practice of cytopathology and ways of improving specimen processing. Clear guidelines or recommendations should be developed on quality control and how pathologists and institutions could integrate and support this activity.

\section{Conflict of Interests}

The author declares no conflict of interests.

\section{References}

[1] G. Bevilacqua, F. Bosman, T. Dassesse et al., "The role of the pathologist in tissue banking. European Consensus Expert Group Report," Virchows Archiv, vol. 456, no. 4, pp. 449-454, 2010.

[2] P. H. J. Riegman, M. M. Morente, F. Betsou, P. de Blasio, and P. Geary, "Biobanking for better healthcare," Molecular Oncology, vol. 2, no. 3, pp. 213-222, 2008.

[3] A. M. Brand and N. M. Probst-Hensch, "Biobanking for epidemiological research and public health," Pathobiology, vol. 74, no. 4, pp. 227-238, 2007.

[4] P. H. J. Riegman, W. N. M. Dinjens, and J. W. Oosterhuis, "Biobanking for interdisciplinary clinical research," Pathobiology, vol. 74, no. 4, pp. 239-244, 2007.

[5] Y. Y. Yu and Z. G. Zhu, "Significance of biological resource collection and tumor tissue bank creation," World Journal of Gastrointestinal Oncology, vol. 15, no. 2, pp. 5-8, 2010.

[6] A. El Hamidi, G. Kocjan, and M. Q. Du, "Clonality analysis of archival cervical smears: correlation of monoclonality with grade and clinical behavior of cervical intraepithelial neoplasia," Acta Cytologica, vol. 47, no. 2, pp. 117-123, 2003.

[7] M. Jarzab, P. Rózanowski, M. Kowalska et al., "Optimization of the method of RNA isolation from paraffin blocks to assess gene expression in breast cancer," Polish Journal of Pathology, vol. 59, no. 2, pp. 85-91, 2008.

[8] D. M. Euhus, A. Maitra, I. I. Wistuba et al., "Use of archival fine-needle aspirates for the allelotyping of tumors," Cancer, vol. 87, no. 6, pp. 372-379, 1999.

[9] K. Canfell, W. Gray, P. Snijders et al., "Factors predicting successful DNA recovery from archival cervical smear samples," Cytopathology, vol. 15, no. 5, pp. 276-282, 2004.

[10] H. Lee, T.-J. Yoon, J.-L. Figueiredo, F. K. Swirski, and R. Weissleder, "Rapid detection and profiling of cancer cells in fine-needle aspirates," Proceedings of the National Academy of Sciences of the United States of America, vol. 106, no. 30, pp. 12459-12464, 2009.
[11] S. Krishnamurthy, "Applications of molecular techniques to fine-needle aspiration biopsy," Cancer, vol. 111, no. 2, pp. 106122, 2007.

[12] R. Mattu, L. Sorbara, A. C. Filie et al., "Utilization of polymerase chain reaction on archival cytologic material: a comparison with fresh material with special emphasis on cerebrospinal fluids," Modern Pathology, vol. 17, no. 10, pp. 1295-1301, 2004.

[13] D. J. Demetrick, "The use of archival frozen tumor tissue imprint specimens for fluorescence in situ hybridization," Modern Pathology, vol. 9, no. 2, pp. 133-136, 1996.

[14] I. Marchetti, F. Lessi, C. M. Mazzanti et al., "A morphomolecular diagnosis of papillary thyroid carcinoma: BRAF V600E detection as an important tool in preoperative evaluation of fine-needle aspirates," Thyroid, vol. 19, no. 8, pp. 837842, 2009.

[15] G. D. Smith, B. E. Chadwick, C. Willmore-Payne, and J. S. Bentz, "Detection of epidermal growth factor receptor gene mutations in cytology specimens from patients with non-small cell lung cancer utilising high-resolution melting amplicon analysis," Journal of Clinical Pathology, vol. 61, no. 4, pp. 487-493, 2008.

[16] S. K. Apple, J. R. Hecht, J. M. Novak, R. K. Nieberg, D. L. Rosenthal, and W. W. Grody, "Polymerase chain reactionbased K-ras mutation detection of pancreatic adenocarcinoma in routine cytology smears," American Journal of Clinical Pathology, vol. 105, no. 3, pp. 321-326, 1996.

[17] M. W. Beaty, Z. Zhuang, W. S. Park et al., "Fine-needle aspiration of metastatic clear cell carcinoma of the kidney: employment of microdissection and the polymerase chain reaction as a potential diagnostic tool," Cancer, vol. 81, no. 3, pp. 180-186, 1997.

[18] M. M. Quezado, A. D. Abati, A. V. Albuquerque, J. Wilson, M. J. Merino, and A. C. Filie, "Morphologic diversity in malignant melanoma: the potential use of microdissection and the polymerase chain reaction for diagnosis," Modern Pathology, vol. 11, no. 10, pp. 1010-1015, 1998.

[19] L. E. Grosso and B. Collins, "DNA polymerase chain reaction using fine needle aspiration biopsy smears to evaluate nonHodgkin's lymphoma," Acta Cytologica, vol. 43, no. 5, pp. 837841, 1999.

[20] M. Kikuchi, K. Kitamura, Y. Nishio et al., "Diagnosis of B-cell lymphoma: utility of the polymerase chain reaction for detecting clonality from archival cytologic smears," Acta Cytologica, vol. 46, no. 2, pp. 349-356, 2002.

[21] B. Garicochea, M. G. Cliquet, N. Melo, A. Del Giglio, P. E. Dorlhiac-Llacer, and D. A. F. Chamone, "Leptomeningeal involvement in chronic lymphocytic leukemia identified by polymerase chain reaction in stored slides: a case report," Modern Pathology, vol. 10, no. 5, pp. 500-503, 1997.

[22] B. G. Bentz, B. T. Miller, J. A. Holden, L. R. Rowe, and J. S. Bentz, "B-RAF V600E mutational analysis of fine needle aspirates correlates with diagnosis of thyroid nodules," Otolaryngology, vol. 140, no. 5, pp. 709-714, 2009.

[23] H. Liu, X. Huang, Y. Zhang et al., "Archival fixed histologic and cytologic specimens including stained and unstained materials are amenable to RT-PCR," Diagnostic Molecular Pathology, vol. 11, no. 4, pp. 222-227, 2002.

[24] T. Štoos-Veić, A. Livun, R. Ajduković, V. Pejša, O. Jakšić, and R. Kušec, "Detection of $t(14 ; 18)$ by PCR of IgH/BCL2 Fusion gene in follicular lymphoma from archived cytological smears," Collegium Antropologicum, vol. 34, no. 2, pp. 425429, 2010. 
[25] M. J. Kube, D. A. McDonald, J. W. Quin, and M. L. Greenberg, "Use of archival and fresh cytologic material for the polymerase chain reaction: detection of the bcl-2 oncogene in lymphoid tissue obtained by fine needle biopsy," Analytical and Quantitative Cytology and Histology, vol. 16, no. 3, pp. 174-182, 1994.

[26] J. Rao, S. K. Apple, Y. Jin, S. Lin, R. K. Nieberg, and S. L. Hirtschowitz, "Comparative polymerase chain reaction analysis of c-myc amplification on archival breast fine-needle aspiration materials," Cancer Epidemiology Biomarkers and Prevention, vol. 9, no. 2, pp. 175-179, 2000.

[27] J. K. Killian, R. L. Walker, M. Suuriniemi et al., "Archival fineneedle aspiration cytopathology (FNAC) samples: untapped resource for clinical molecular profiling," Journal of Molecular Diagnostics, vol. 12, no. 6, pp. 739-745, 2010.

[28] L. Boldrini, S. Gisfredi, S. Ursino et al., "Mutational analysis in cytological specimens of advanced lung adenocarcinoma: a sensitive method for molecular diagnosis," Journal of Thoracic Oncology, vol. 2, no. 12, pp. 1086-1090, 2007.

[29] S. Billah, J. Stewart, G. Staerkel, S. Chen, Y. Gong, and M. Guo, "EGFR and KRAS mutations in lung carcinoma: molecular testing by using cytology specimens," Cancer Cytopathology, vol. 119, no. 2, pp. 111-117, 2011.

[30] K. M. Denning, P. C. Smyth, S. F. Cahill et al., "A molecular expression signature distinguishing follicular lesions in thyroid carcinoma using preamplification RT-PCR in archival samples," Modern Pathology, vol. 20, no. 10, pp. 1095-1102, 2007.

[31] A. R. Tomás, M. J. Praça, R. Fonseca, S. André, and E. Mendonça, "Assessing HER-2 status in fresh frozen and archival cytological samples obtained by fine needle aspiration cytology," Cytopathology, vol. 15, no. 6, pp. 311-314, 2004.

[32] D. A. Dillon, E. Hipolito, K. Zheng, D. L. Rimm, and J. C. Costa, "p53 mutations as tumor markers in fine needle aspirates of palpable breast masses," Acta Cytologica, vol. 46, no. 5, pp. 841-847, 2002.

[33] S. Alkan, I. A. Eltoum, S. Tabbara, E. Day, and D. S. Karcher, "Usefulness of molecular detection of human herpesvirus-8 in the diagnosis of Kaposi sarcoma by fine-needle aspiration," American Journal of Clinical Pathology, vol. 111, no. 1, pp. 9196, 1999.

[34] L. Vago, P. Zerbi, R. Caldarelli-Stefano et al., "Polymerase chain reaction for Mycobacterium tuberculosis complex DNA: use on negative archival Ziehl-Neelsen cytologic samples," Acta Cytologica, vol. 44, no. 6, pp. 1023-1028, 2000.

[35] N. Gupta, K. Sharma, A. Barwad et al., "Thyroid tuberculosis-role of PCR in diagnosis of a rare entity," Cytopathology. In press.

[36] R. Sahoo, A. Banerjee, K. Payal et al., "Improved method for detection of methylation status of genes from limited, archived, FFPE and FNAC samples," Journal of Clinical and Diagnostic Research, vol. 3, no. 3, pp. 1493-1499, 2009.

[37] P. A. Fetsch, N. L. Simone, P. K. Bryant-Greenwood et al., "Proteomic evaluation of archival cytologic material using SELDI affinity mass spectrometry: potential for diagnostic applications," American Journal of Clinical Pathology, vol. 118, no. 6, pp. 870-876, 2002.

[38] R. F. Ozols, R. S. Herbst, Y. L. Colson et al., "Clinical cancer advances 2006: major research advances in cancer treatment, prevention, and screening - a report from the American Society of Clinical Oncology," Journal of Clinical Oncology, vol. 25, no. 1, pp. 146-162, 2007. 


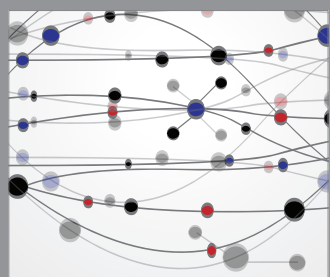

The Scientific World Journal
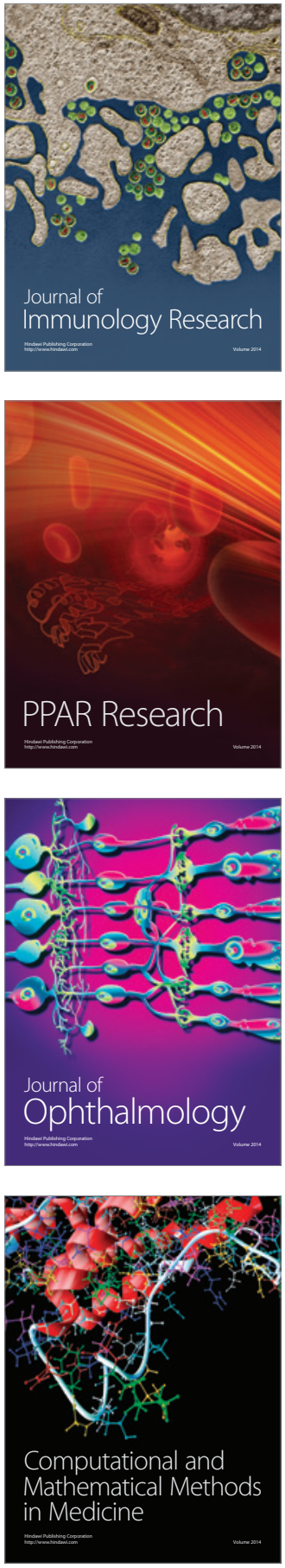

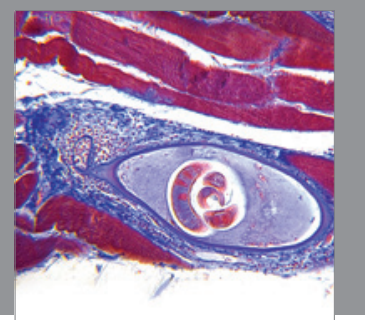

Gastroenterology

Research and Practice
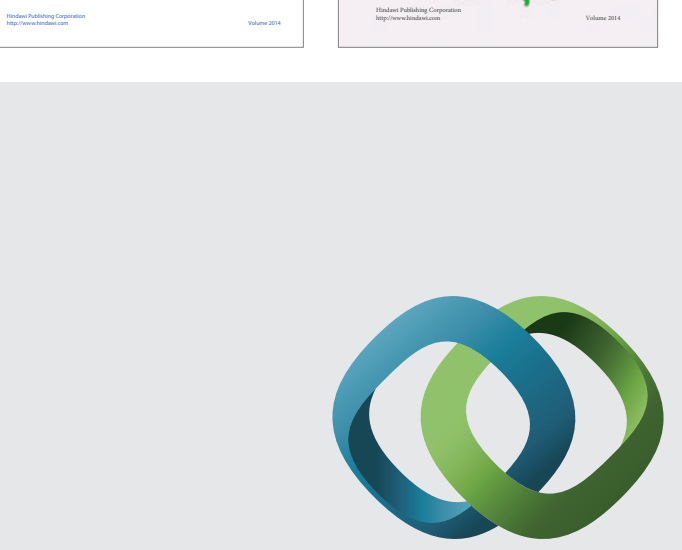

\section{Hindawi}

Submit your manuscripts at

http://www.hindawi.com
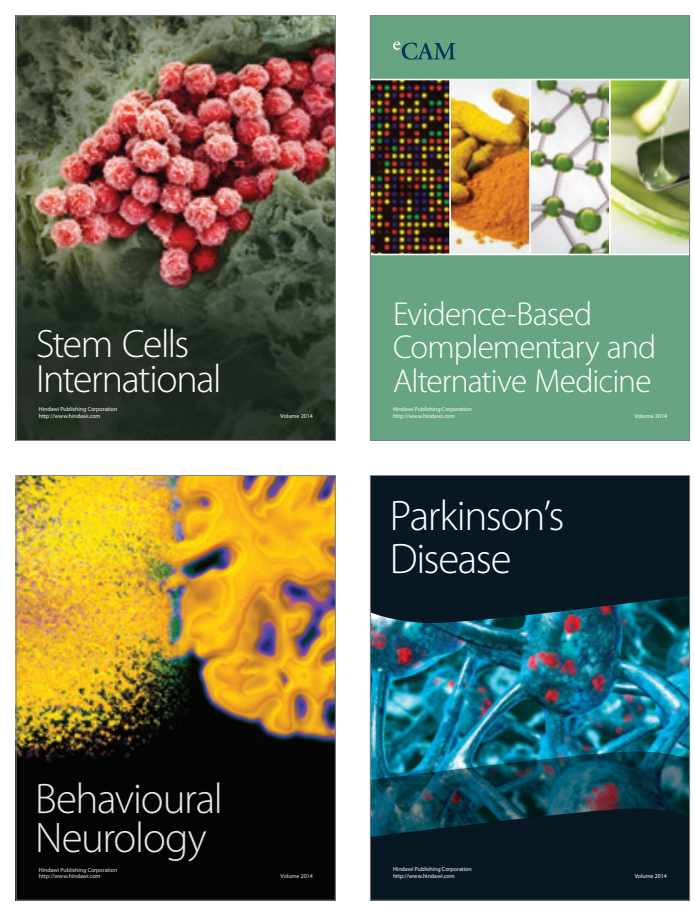

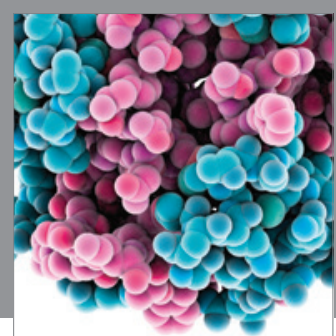

Journal of
Diabetes Research

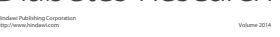

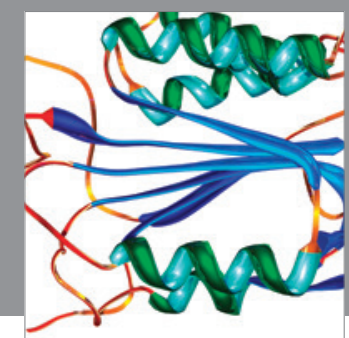

Disease Markers
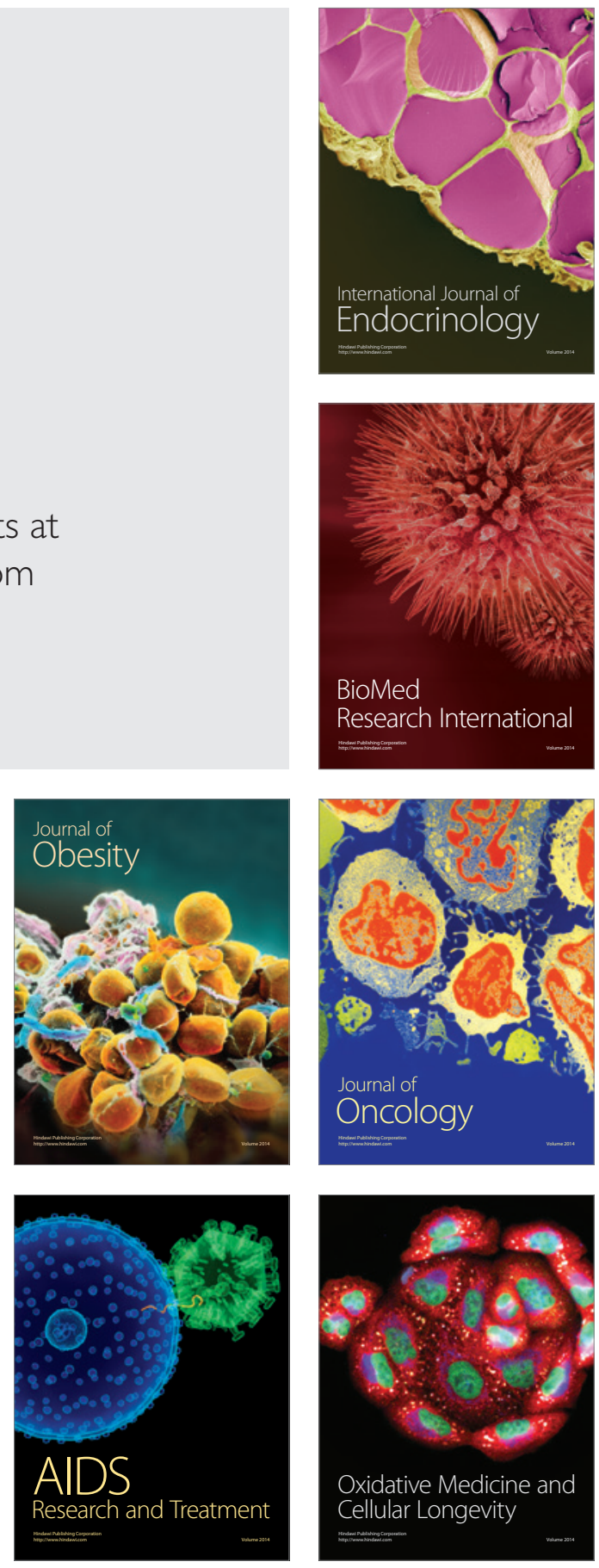